Canad. Math. Bull. Vol. 23 (2), 1980

\title{
SMOOTHNESS PROPERTIES OF BOUNDED SOLUTIONS OF DIRICHLET'S PROBLEM FOR ELLIPTIC EQUATIONS IN REGIONS WITH CORNERS ON THE BOUNDARY
}

\author{
A. AZZAM
}

We study here the smoothness of solutions of the Dirichlet problem for elliptic equations in a region $G$ with a piece-wise smooth boundary. The smoothness of the solution given depends on the smoothness of the coefficients of the equation, the boundary, the boundary function and the values of the angles on the boundary and the values of the coefficients of the second derivatives at the corners.

$\S 1$. Introduction. Boundary value problems for the linear second order elliptic equation

$$
\sum_{i, j=1}^{2} a_{i j}\left(x_{1}, x_{2}\right) \frac{\partial^{2} u}{\partial x_{i} \partial x_{i}}+\sum_{i=1}^{2} a_{i}\left(x_{1}, x_{2}\right) \frac{\partial u}{\partial x_{i}}+a\left(x_{1}, x_{2}\right) u=f\left(x_{1}, x_{2}\right)
$$

in a domain with smooth boundary have been thoroughly investigated. In the works of Agmon, Douglis, Nirenberg [1] and Browder [2], the normal solvability of such problems for general boundary conditions, satisfying the SapiroLopatinskii condition has been established. It was also proven that, if the right hand side of (1.1), the coefficients of the equation, the boundary and the boundary operators are infinitely differentiable, then the solution of the problem is also infinitely differentiable (see also [4], [7], [10]). If the boundary contains a corner, this is no longer true. The reason is that, it is not possible in this case to smooth the boundary by means of a smooth transformation. Moreover, from the simplest examples, it is apparent that when the boundary contains angular points the solution of the problem may not be infinitely differentiable for infinitely differentiable right hand side, coefficients and boundary functions. In $[5,6]$ Kondratev considered some special Sobolev spaces with weight functions. In these spaces, he studied general boundary value problems for equation (1.1) in a domain whose boundary is piece-wise smooth. We study here the first boundary value problem for equation (1.1) in the space $C_{m+\alpha}$.

Received by the editors May 3, 1978 and, in revised form, January 5, 1979. 
Definitions 1.1. The function $g\left(x_{1}, x_{2}\right)$ is said to belong to $C_{m+\alpha}(G) m \geq 0$ an integer, $0<\alpha<1$, if $g\left(x_{1}, x_{2}\right)$ has $m$ continuous derivatives in $G$, and the derivatives of order $m$ satisfy in $G$ a Hölder condition with exponent $\alpha$.

1.2. The Hölder coefficient of the derivatives of order $k$ of $g\left(x_{1}, x_{2}\right)$ in the domain $G$ is defined as

$$
H_{\alpha}^{G}\left(D^{k} g\right)=\text { l.u.b. } \frac{\left|D^{k} g(P)-D^{k} g(Q)\right|}{(\overline{P Q})^{\alpha}}
$$

where l.u.b. is over $P \neq Q$ in $G$, and all the derivatives of order $k$.

1.3. In $C_{m+\alpha}(G)$, the norms may be defined as follows

$$
\begin{aligned}
\|g\|_{0} & =\max _{G}\left|g\left(x_{1}, x_{2}\right)\right| \\
\|g\|_{k+\alpha} & =\sum_{i=0}^{k}\left\|D^{i} g\right\|_{0}+H_{\alpha}^{G}\left(D^{k} g\right), \quad k=0,1, \ldots, m .
\end{aligned}
$$

In [1], it is proved that, if the right hand side and the coefficients of equation (1.1) are of class $C_{m+\alpha}(\bar{G})$, and if the boundary $\Gamma$ of $G$ can be represented parametrically by functions of class $C_{m+2+\alpha}$, and if the boundary function $\phi$ belongs to $C_{m+2+\alpha}(\Gamma)$, then any solution of the Dirichlet problem

$$
\begin{gathered}
\sum_{i, j=1}^{2} a_{i j}\left(x_{1}, x_{2}\right) \frac{\partial^{2} u}{\partial x_{i} \partial x_{j}}+\sum_{i=1}^{2} a_{i}\left(x_{1}, x_{2}\right) \frac{\partial u}{\partial x_{i}}+a\left(x_{1}, x_{2}\right) u=f\left(x_{1}, x_{2}\right) \\
u=\phi \text { on } \Gamma
\end{gathered}
$$

belongs to $C_{m+2+\alpha}(\bar{G})$. If the boundary $\Gamma$ contains some corners, and the open arcs between these corners are of class $C_{m+2+\alpha}$, then the solution $u\left(x_{1}, x_{2}\right)$ belongs to $C_{m+2+\alpha}\left(G_{1}\right) \cap C_{0}(\bar{G})$ where $G_{1}$ is any compact subdomain of $\bar{G}$ with positive distances from the corners.

In a rectangle $G=\left\{\left(x_{1}, x_{2}\right), 0<x_{1}<a, 0<x_{2}<b\right\}$, Nikolskii [11] studied the problem

$$
\begin{gathered}
\Delta u \equiv \frac{\partial^{2} u}{\partial x_{1}^{2}}+\frac{\partial^{2} u}{\partial x_{2}^{2}}=0 \text { in } G \\
u=\phi \text { on } \Gamma .
\end{gathered}
$$

He gave necessary and sufficient conditions for the solution to belong to $C_{m+2+\alpha}(\bar{G})$. These conditions are that on the open intervals $0<x_{1}<a, 0<x_{2}<$ $b$ the boundary function $\phi$ belongs to $C_{m+2+\alpha}$ and at the four corners it satisfies certain compatibility conditions. These results were generalized in [3] by Fufaev to the case of Poisson equation. Fufaev also proved that if the angles at the corners are of values $\pi / q, q=2,3, \ldots$ then the solution of the Dirichlet 
problem

$$
\begin{gathered}
\Delta u=f\left(x_{1}, x_{2}\right) \text { in } G \\
u=0 \text { on } \Gamma
\end{gathered}
$$

will belong to $C_{m+2+\alpha}(\bar{G})$ if and only if $f\left(x_{1}, x_{2}\right) \varepsilon C_{m+\alpha}(\bar{G})$ and at the corners $f\left(x_{1}, x_{2}\right)$ satisfies certain agreement conditions (see also [13]). In domains with angles $\pi / q, q=2,3, \ldots$ Volkov [14] studied mixed boundary value problems for the Laplace equation, and gave the necessary and sufficient conditions for the solution to belong to $C_{m+2+\alpha}(\bar{G})$. If the angle $\omega$ on the boundary is not of the form $\pi / q, q=2,3, \ldots$ then the solution of problem (1.4)-(1.5) may not belong to $C_{m+2+\alpha}(\bar{G})$. Fufaev [3] proved that if $\pi / \omega$ is not an integer, then the smaller the angle $\omega$ the smoother in $\bar{G}$ is the solution $u$ of problem (1.4) (1.5). He proved that if $(\pi / \omega)>m+2+\alpha$, then $u\left(x_{1}, x_{2}\right) \varepsilon C_{m+2+\alpha}(\bar{G})$ provided that the boundary function is continuous on the whole boundary $\Gamma$ and is of class $C_{m+2+\alpha}$ on the open intervals between the corners. In this paper we generalize this result to the case of problem (1.2)-(1.3). We first prove this result in a circular sector then we use this to prove the theorem in the general case.

$\S 2$. The problem in a circular sector. Consider the sector $\Omega_{\sigma}=\{(r, \theta)$, $r \leq \sigma<1, \beta<\theta<\omega+\beta\}$ where $(r, \theta)$ the polar coordinates of the point $x=$ $\left(x_{1}, x_{2}\right)$ and $(\pi / \omega)>m+2+\alpha$ and $\beta>0$ satisfies $\pi /(\omega+2 \beta)>m+2+\alpha$. Suppose that the function $w\left(x_{1}, x_{2}\right)$ satisfies inside $\Omega_{\sigma}$ the elliptic equation

$$
L w \equiv \sum_{i, j=1}^{2} b_{i j}(x) \frac{\partial^{2} w}{\partial x_{i} \partial x_{j}}+\sum_{i=1}^{2} b_{i}(x) \frac{\partial w}{\partial x_{i}}+b(x) w=g(x)
$$

where $g(x), b_{i j}(x), b_{i}(x)$ and $b(x)$ belong to $C_{m+\alpha}\left(\bar{\Omega}_{\sigma}\right)$ and $b_{i j}(0,0)=\delta_{i j}$ the kronecker delta, $i, j=1,2$. Suppose also that $w(x)$ is bounded in $\bar{\Omega}_{\sigma}$ and that its boundary value $\Psi(r, \theta)$ on the two lines $\theta=\beta$ and $\theta=\omega+\beta$ belongs to $C_{m+2+\alpha}, 0 \leq r<\sigma$, and

$$
\left.\Psi^{(k)}(0, \theta) \equiv \frac{d^{k} \Psi}{d r^{k}}\right|_{r=0}=0, \quad k=0,1, \ldots, m+2
$$

We also assume that $g(x)$ satisfies

$$
\left.D^{k} g(0,0) \equiv \frac{\partial^{k} g}{\partial x_{1}^{k_{1}} \partial x_{2}^{k-k_{1}}}\right|_{(0,0)}=0, \quad k=0,1, \ldots, m
$$

for all $k_{1}=0,1, \ldots, k$.

THEOREM 1. There exists a number $r_{0}, 0<2 r_{0}<\sigma$ such that in $\Omega_{2 r_{0}}$ the bounded solution $w(x)$ of the problem

$$
\begin{aligned}
L w & =g(x) \text { in } \Omega_{\sigma} \\
w & =\Psi \text { on } \Gamma
\end{aligned}
$$


where $g$ and $\psi$ fulfill (2.2) and (2.3), satisfies the inequality

$$
|w| \leq M r^{m+2+\alpha}
$$

In the course of the proof we will need the following two lemmas.

LEMMA 1 [7]. Let $\Omega$ be a finite domain with diameter $r$, and let $L$ be a linear second order elliptic operator. Let $v \in C_{2}(\Omega)$ and $L v \geq 0(\leq 0)$ inside $\Omega$, while $v \leq 0(\geq 0)$ on the boundary of $\Omega$. There exists $r_{0}>0$, such that if $r \leq r_{0}$, then $v \leq 0(\geq 0)$ will be satisfied inside $\Omega$.

LEMMA 2 [1]. Let $\Gamma^{\prime}$ be a portion of the boundary $\Gamma$ of a domain $\Omega$, and $\Gamma^{\prime} \in C_{m+2+\alpha}$. Let $\Omega^{\prime}$ be a subdomain of $\Omega$ with the property that the intersection of the boundary of $\Omega^{\prime}$ with $\Gamma$ lies in the interior of $\Gamma^{\prime}$, then any bounded solution $w(x)$ of the problem (2.4)-(2.5) in $\Omega$ will satisfy Schauder's inequality

$$
\|w\|_{m+2+\alpha}^{\Omega^{\prime}} \leq \delta\left(\|w\|_{0}^{\Omega}+\|g\|_{m+\alpha}^{\Omega}+\|\Psi\|_{m+2+\alpha}^{\Gamma^{\prime}}\right)
$$

where $\delta>0$ is a finite number independent of $w$.

We now prove Theorem 1 .

Proof. Since on the two lines $\theta=\beta$ and $\theta=\omega+\beta$ we have $\Psi \in C_{m+2+\alpha}$ then from (2.2) we get

$$
\begin{aligned}
& \left|\Psi^{(m+2)}(r, \theta)\right|=\left|\Psi^{(m+2)}(r, \theta)-\Psi^{(m+2)}(0, \theta)\right| \leq K_{m+2} r^{\alpha} \\
& \left|\Psi^{(m+1)}(r, \theta)\right| \leq \int_{0}^{r}\left|\Psi^{(m+2)}(r, \theta)\right| d \theta \leq K_{m+1} r^{1+\alpha}
\end{aligned}
$$

And generally for any $k=0,1, \ldots, m+2$

$$
\left|\Psi^{(k)}(r, \theta)\right| \leq K_{k} r^{m+2-k+\alpha}
$$

Similary for $g\left(x_{1}, x_{2}\right)$

$$
\left|D^{k} g\left(x_{1}, x_{2}\right)\right| \leq H_{k} r^{m-k+\alpha} \quad k=0,1, \ldots, m
$$

Consider now the function $v(x)=-M r^{\nu} \sin \lambda \theta$ where $M>0$ will be defined later and $\lambda=\pi /(\omega+2 \beta)>m+2+\alpha=\nu$. We rewrite the operator $L w$ as follows

$$
L w=\frac{\partial^{2} w}{\partial x_{1}^{2}}+\frac{\partial^{2} w}{\partial x_{2}^{2}}+\sum_{i, j=1}^{2}\left[b_{i j}(x)-\delta_{i j}\right] \frac{\partial^{2} w}{\partial x_{i} \partial x_{j}}+\sum_{i=1}^{2} b_{i}(x) \frac{\partial w}{\partial x_{i}}+b(x) w
$$

In particular

$$
L v=M\left(\lambda^{2}-v^{2}\right) r^{m+\alpha} \sin \lambda \theta+M \sum_{i, j=1}^{2}\left[b_{i j}(x)-\delta_{i j}\right] H_{i j} r^{m+\alpha}
$$


where $H_{i j}$ and $h_{i}$ are bounded functions, say

$$
\sum_{i, j=1}^{2}\left|H_{i j}\right|+\sum_{i=1}^{2}\left|h_{i}\right| \leq R .
$$

Since $b_{i j}\left(x_{1}, x_{2}\right)$ are continuous functions and $b_{i j}(0,0)=\delta_{i j}$, then for any $\varepsilon>0$, we can find $r_{0}>0$ such that if $r \leq 2 r_{0}$,

$$
\left|b_{i j}(x)-\delta_{i j}\right|<\varepsilon / 4 R \quad i, j=1,2 .
$$

Since for $\beta<\theta<\omega+\beta, \sin \lambda \theta \geq \sin \lambda \beta$, then for $r \leq 2 r_{0}$, (2.11) gives

$$
\begin{aligned}
L v & \geq M\left[\left(\lambda^{2}-\nu^{2}\right) \sin \lambda \beta-\varepsilon\right] r^{m+\alpha}-2 M R r^{m+1+\alpha} \\
& \geq M\left[\left(\lambda^{2}-\nu^{2}\right) \sin \lambda \beta-\varepsilon-4 R r_{0}\right] r^{m+\alpha} .
\end{aligned}
$$

We choose $\varepsilon>0$ sufficiently small, such that $A=\left(\lambda^{2}-\nu^{2}\right) \sin \lambda \beta-\varepsilon>0$ then we take $r_{0}$ small enough to make $B=A-4 R r_{0}>0$ then we set $M>H_{0} / B$, where $H_{0}$ is taken from (2.9). Thus inside $\Omega_{2 r_{0}}$ we have $L v \geq H_{0} r^{m+\alpha} \geq g(x)$, i.e. $L(w-v)=L w-L v \leq 0$.

We now choose $M$ sufficiently large, such that on the boundary of $\Omega_{2 r_{0}}$, $w-v \geq 0$. On the two lines $\theta=\beta$ and $\theta=\omega+\beta$ we have

$$
w-v=\Psi(r, \theta)+M r^{m+2+\alpha} \sin \lambda \beta
$$

and from (2.8) we get

$$
w-v \geq\left(M \sin \lambda \beta-K_{0}\right) r^{m+2+\alpha} .
$$

On the arc $r=2 r_{0}$ we have

$$
w-v \geq w+M\left(2 r_{0}\right)^{m+2+\alpha} \sin \lambda \beta \geq-\|w\|_{0}^{\Omega_{\sigma}}+M\left(2 r_{0}\right)^{m+2+\alpha} \sin \lambda \beta
$$

We now take $M$ sufficiently large so that the right hand sides of (i) and (ii) are nonnegative. Now inside $\Omega_{2 r_{0}}, L(w-v) \leq 0$ while on its boundary $w-v \geq 0$. We take $r_{0}$ sufficiently small, such that in $\Omega_{2 r_{0}}$ we can apply lemma 1 . Thus in $\Omega_{2 r_{0}}$ we now have $w-v \geq 0$, i.e.

$$
w \geq-M r^{m+2+\alpha} \sin \lambda \theta \geq-M r^{m+2+\alpha} .
$$

Similarly, taking $r_{0}$ sufficiently small and $M$ sufficiently large we can prove that in $\Omega_{2 r_{0}}, w \leq M r^{m+2+\alpha}$ i.e. in $\Omega_{2 r_{1}}$

$$
|w| \leq M r^{m+2+\alpha} \text {. }
$$

This proves the theorem.

THEOREM 2. Under the assumptions of Theorem 1, the following inequalities hold in $\Omega_{r_{0}}$

$$
\left|D^{k} w\right| \leq M_{k} r^{m+2-k+\alpha}, \quad k=0,1, \ldots, m+2
$$

where $D^{k} w$ is any derivative of order $k$ of $w(x)$. 
Proof. Consider the domains

$$
\begin{aligned}
& \Omega_{n}=\left\{(r, \theta) ; \frac{r_{0}}{2^{n+1}} \leq r \leq \frac{r_{0}}{2^{n}} ; \beta \leq \theta \leq \omega+\beta\right\} \\
& \Omega_{n}^{\prime}=\Omega_{n-1} \cup \Omega_{n} \cup \Omega_{n+1} .
\end{aligned}
$$

We denote by $\Gamma_{n}^{\prime}$ the straight parts of the boundary of $\Omega_{n}^{\prime}$. Any point $(r, \theta) \in \Omega_{r_{0}}$ belongs to some $\Omega_{n}$ with $\Omega_{n}^{\prime} \subset \Omega_{2 r_{0}}$. The transformation

$$
x_{i}=\frac{1}{2^{n}} y_{i} \quad i=1,2
$$

transforms $\Omega_{n}$ and $\Omega_{n}^{\prime}$ to $\Omega_{0}$ and $\Omega_{0}^{\prime}$ respectively. In $\Omega_{0}^{\prime}$ the function $w_{0}(y)=$ $w_{0}\left(y_{1}, y_{2}\right)=w\left(y_{1} / 2^{n}, y_{2} / 2^{n}\right)$ satisfies the elliptic equation

$$
\sum_{i, j=1}^{2} c_{i j}(y) \frac{\partial^{2} w_{0}}{\partial y_{i} \partial y_{j}}+2^{-n} \sum_{i=1}^{2} c_{i}(y) \frac{\partial w_{0}}{\partial y_{i}}+2^{-2 n} c(y) w_{0}=2^{-2 n} g_{0}(y)
$$

where $c_{i j}(y)=b_{i j}\left(y / 2^{n}\right)$ and similarly the functions $c_{i}(y), c(y)$ and $g_{0}(y)$ may be defined in terms of $b_{i}, b$ and $g$. The boundary value of $w_{0}(y)$ is $\Psi_{0}(\rho, \theta)=$ $\Psi\left(\rho / 2^{n}, \theta\right) ; \rho^{2}=y_{1}^{2}+y_{2}^{2}$. In $\Omega_{0}$ and $\Omega_{0}^{\prime}$ the Schauder's inequality (2.7) gives

$$
\left\|w_{0}\right\|_{m+2+\alpha}^{\Omega_{0}} \leq \delta\left(\left\|w_{0}\right\|_{0}^{\Omega_{0}^{\prime}}+2^{-2 n}\left\|g_{0}\right\|_{m^{\prime}+\alpha}^{\Omega_{0}^{\prime}}+\left\|\Psi_{0}\right\|_{m^{\prime}+2+\alpha}^{\Gamma_{0}^{\prime}}\right)
$$

Now $\left\|w_{0}\right\|_{0^{\circ}}^{\Omega_{0}}=\|w\|_{0^{n}}^{\Omega^{\prime}}$ and from (2.6) it follows that

$$
\left\|w_{0}\right\|_{0}^{\Omega_{0}^{\prime}} \leq M r^{m+2+\alpha} \leq T_{1}\left(\frac{1}{2^{n}}\right)^{m+2+\alpha}
$$

Since $g_{0}(y)=g\left(y / 2^{n}\right)$, then

$$
D_{0}^{k} g_{0}(y)=\left(\frac{1}{2^{n}}\right)^{k} D^{k} g(x) \quad k=0,1,2, \ldots, m
$$

where $D_{0}^{k}$ is the derivative in the $y$-plane corresponding to $D^{k}$. From that and from (2.9) we get

$$
\left|D_{0}^{k} g_{0}(y)\right| \leq H\left(\frac{1}{2^{n}}\right)^{m+\alpha} \quad k=0,1, \ldots, m
$$

If $P$ and $Q$ are any two points in $\Omega_{n}^{\prime}$ and their images in $\Omega_{0}^{\prime}$ are $P_{0}$ and $Q_{0}$, then

$$
\frac{D_{0}^{m} g_{0}\left(P_{0}\right)-D_{0}^{m} g_{0}\left(Q_{0}\right)}{\left(\overline{P_{0} Q_{0}}\right)^{\alpha}}=\left(\frac{1}{2^{n}}\right)^{m+\alpha} \frac{D^{m} g(P)-D^{m} g(Q)}{(\overline{P Q})^{\alpha}}
$$

From this it follows that

$$
H_{\alpha}^{\Omega_{\mathrm{o}}^{\prime}}\left(D_{0}^{m} g_{0}\right)=\left(\frac{1}{2^{n}}\right)^{m+\alpha} H_{\alpha}^{\Omega_{n}^{\prime}}\left(D^{m} g\right)
$$


Thus

$$
\begin{aligned}
\left\|g_{0}\right\|_{m+\alpha}^{\Omega_{0}^{\prime}} & =\sum_{k=0}^{m}\left\|D_{0}^{k} g_{0}\right\|_{0}^{\Omega_{0}^{\prime}}+H_{\alpha}^{\Omega_{o}^{\prime}}\left(D_{0}^{m} g_{0}\right) \leq T\left(\frac{1}{2^{n}}\right)^{m+\alpha}\|g\|_{m^{\prime}+\alpha}^{\Omega_{n}^{\prime}} \\
& \leq T_{2}\left(\frac{1}{2^{n}}\right)^{m+\alpha}
\end{aligned}
$$

Similarly we can show that

$$
\left\|\Psi_{0}\right\|_{m+2+\alpha}^{\Gamma_{0}^{\prime}} \leq T_{3}\left(\frac{1}{2^{n}}\right)^{m+2+\alpha}
$$

Thus (2.15) yields

$$
\begin{aligned}
\left\|w_{0}\right\|_{m+2+\alpha}^{\Omega_{0}} & \leq \delta\left[T_{1}\left(\frac{1}{2^{n}}\right)^{m+2+\alpha}+T_{2}\left(\frac{1}{2^{n}}\right)^{m+2+\alpha}+T_{3}\left(\frac{1}{2^{n}}\right)^{m+2+\alpha}\right] \\
& =\delta_{0}\left(\frac{1}{2^{n}}\right)^{m+2+\alpha}
\end{aligned}
$$

Since $\left|D_{0}^{k} w_{0}\right| \leq\left\|w_{0}\right\|_{m+2+\alpha}^{\Omega_{0}}, k=0,1, \ldots, m+2$ and since $D_{0}^{k} w_{0}=\left(1 / 2^{n}\right)^{k} D^{k} w$, we get that in $\Omega_{n}$

$$
\left(\frac{1}{2^{n}}\right)^{k}\left|D^{k} w\right| \leq \delta_{0}\left(\frac{1}{2^{n}}\right)^{m+2+\alpha}
$$

or equivalently

$$
\left|D^{k} w\right| \leq \delta_{0}\left(\frac{1}{2^{n}}\right)^{m+2-k+\alpha} \leq M_{k} r^{m+2-k+\alpha}, \quad k=0,1, \ldots, m+2 .
$$

This proves the theorem.

THEOREM 3. Under the assumptions of Theorem $1, w\left(x_{1}, x_{2}\right) \in C_{m+2+\alpha}\left(\Omega_{r_{0}}\right)$.

Proof. Consider any two points $P\left(r_{1}, \theta_{1}\right)$ and $Q\left(r_{2}, \theta_{2}\right)$ in $\bar{\Omega}_{r_{0}}$, and suppose that $0 \leq r_{2} \leq r_{1} \leq r_{0}$. We have two cases to consider

1. $r_{2} \leq \frac{1}{2} r_{1}$. In this case $\overline{P Q} \geq \frac{1}{2} r_{1}$ and

$$
\begin{aligned}
\left|D^{k} w(P)-D^{k} w(Q)\right| & \leq M_{k} r_{1}^{m+2-k+\alpha}+M_{k} r_{2}^{m+2-k+\alpha} \\
& <2 M_{k} r_{1}^{m+2-k+\alpha} ; \quad k=0,1, \ldots, m+2
\end{aligned}
$$

and

$$
\frac{\left|D^{m+2} w(P)-D^{m+2} w(Q)\right|}{(\overline{P Q})^{\alpha}} \leq \frac{2 M_{m+2} r_{1}^{\alpha}}{\left(\frac{1}{2} r_{1}\right)^{\alpha}}=\text { const. }
$$

2. $r_{2}>\frac{1}{2} r_{1}$. We will prove that $w \in C_{m+2+\alpha}\left(G_{0}\right)$, where $G_{0}=\{(r, \theta)$, 
$\left.\left(r_{1} / 2\right) \leq r \leq r_{1}, \beta \leq \theta \leq \omega+\beta\right\}$. The transformation

$$
x_{i}=\frac{2 r_{1}}{r_{0}} y_{i} \quad i=1,2
$$

transforms $G_{0}$ to

$$
G_{1}=\left\{(\rho, \theta), \frac{r_{0}}{4} \leq \rho \leq \frac{r_{0}}{2}, \beta \leq \theta \leq \omega+\beta\right\}, \rho^{2}=y_{1}^{2}+y_{2}^{2}
$$

and transforms $P$ and $Q$ to $P_{1}\left(\rho_{1}, \theta_{1}\right)$ and $Q_{1}\left(\rho_{2}, \theta_{2}\right)$ where $\rho_{1}=r_{0} / 2$ and $\rho_{2}=\left(r_{0} r_{2} / 2 r_{1}\right)>\left(r_{0} / 4\right)$. In

$$
G_{1}^{\prime}=\left\{(\rho, \theta) ; \frac{r_{0}}{8} \leq \rho \leq r_{0}, \beta \leq \theta \leq \omega+\beta\right\}
$$

the function

$$
w_{1}(y)=w_{1}\left(y_{1}, y_{2}\right)=w\left(\frac{2 r_{1}}{r_{0}} y_{1}, \frac{2 r_{1}}{r_{0}} y_{2}\right)
$$

satisfies the elliptic equation

$$
\sum_{i, j=1}^{2} d_{i j}(y) \frac{\partial^{2} w_{1}}{\partial y_{i} \partial y_{j}}+\left(\frac{2 r_{1}}{r_{0}}\right) \sum_{i=1}^{2} d_{i}(y) \frac{\partial w_{1}}{\partial y_{i}}+\left(\frac{2 r_{1}}{r_{0}}\right)^{2} d(y) w_{1}=\left(\frac{2 r_{1}}{r_{0}}\right)^{2} g_{1}(y)
$$

where

$$
d_{i j}(y)=d_{i j}\left(y_{1}, y_{2}\right)=b_{i j}\left(\frac{2 r_{1}}{r_{0}} y_{1}, \frac{2 r_{1}}{r_{0}} y_{2}\right) \text {. }
$$

Similarly the functions $d_{i}(y), d(y)$ and $g_{1}(y)$ can be defined. On the straight parts of the boundary of $G_{1}^{\prime}$, the boundary value of $W_{1}(y)$ is $\Psi_{1}(\rho, \theta)=$ $\Psi\left[\left(2 r_{1} / r_{0}\right) \rho, \theta\right]$. Schauder's inequality $(2.7)$ in $G_{1}$ and $G_{1}^{\prime}$ yields

$$
\left\|w_{1}\right\|_{m+2+\alpha}^{G_{1}} \leq \delta\left[\left\|w_{1}\right\|_{0}^{G_{1}^{\prime}}+\left(\frac{2 r_{1}}{r_{0}}\right)^{2}\left\|g_{1}\right\|_{m+\alpha}^{G_{i}^{\prime}}+\left\|\Psi_{1}\right\|_{m^{1}+2+\alpha}^{\Gamma_{1}^{\prime}}\right]
$$

Exactly as in the proof of Theorem 2 we get

$$
\left\|w_{1}\right\|_{m+2+\alpha}^{G_{1}} \leq \delta_{0} r_{1}^{m+2+\alpha} \text {. }
$$

We note as before that $D_{1}^{k} w_{1}=\left(2 r_{1} / r_{0}\right)^{k} D^{k} w$ where $D_{1}^{k}$ is the derivative corresponding to $D^{k}$, and noting that $\left\|w_{1}\right\|_{\mu} \leq\left\|w_{1}\right\|_{m+2+\alpha}, 0 \leq \mu \leq m+2+\alpha$ we get that in $G_{0}$.

$$
\left(\frac{2 r_{1}}{r_{0}}\right)^{k}\left|D^{k} w\right| \leq \delta_{1} r_{1}^{m+2+\alpha}
$$

or equivalently

$$
\left|D^{k} w\right| \leq N_{k} r_{1}^{m+2-k+\alpha} \quad k=0,1,2, \ldots, m+2 .
$$

Also noting that $H_{\alpha}^{G_{1}}\left(D_{1}^{m+2} w_{1}\right)=\left(2 r_{1} / r_{0}\right)^{m+2+\alpha} H_{\alpha}^{G_{o}}\left(D^{m+2} w\right)$ we get 
$\left(2 r_{1} / r_{0}\right)^{m+2+\alpha} H_{\alpha}^{G_{o}}\left(D^{m+2} w\right) \leq \delta_{1} r_{1}^{m+2+\alpha}$

$$
\text { i.e) } H_{\alpha}^{G_{o}}\left(D^{m+2} w\right) \leq H \text {. }
$$

This means that $w \in C_{m+2+\alpha}\left(G_{0}\right)$. The theorem is thus proved.

$\S 3$. The general case. Let the domain $G$ have a boundary $\Gamma$ consisting of a piece of a smooth curve of class $C_{m+2+\alpha}$ the ends of which join at the point 0 forming an angle $\gamma, 0<\gamma<2 \pi$. In $G$ we consider the first boundary value problem

$$
\begin{aligned}
\sum_{i, j=1}^{2} a_{i j}(x) \frac{\partial^{2} u}{\partial x_{i} \partial x_{j}}+\sum_{i=1}^{2} a_{i}(x) \frac{\partial u}{\partial x_{i}}+a(x) u=f(x) \\
u=\phi \text { on } \Gamma
\end{aligned}
$$

the right hand side of (3.1) and the coefficients $a_{i j}, a_{i}$ and $a$ belong to $C_{m+\alpha}(\bar{G})$. The boundary function $\phi$ is continuous on $\Gamma$ and is of class $C_{m+2+\alpha}(\Gamma \backslash\{0\})$. We transform the equation

$$
\sum_{i, j=0}^{2} a_{i j}(0) \frac{\partial^{2} u}{\partial x_{i} \partial x_{j}}=0
$$

to canonical form. The new angle after transformation $\omega(0)$ is independent of the transformation used and is given by

$$
\omega(0)=\arctan \frac{\left[a_{11}(0) a_{22}(0)-a_{12}^{2}(0)\right]^{1 / 2}}{a_{22}(0) \cot \gamma-a_{12}(0)}
$$

THEOREM 4. If $(\pi / \omega(0))>m+2+\alpha$, then the bounded solution of problem (3.1)-(3.2) where $f$ belongs to $C_{m+\alpha}(\bar{G})$ and $\phi$ belongs to $C_{m+2+\alpha}(\Gamma \backslash\{0\}) \cap C_{0}(\Gamma)$ belongs to $C_{m+2+\alpha}(\bar{G})$.

Proof. It is sufficient to prove the theorem in any neighborhood $G_{0} \subset \bar{G}$, of the corner 0 since $u(x) \in C_{m+2+\alpha}\left(\bar{G} \backslash G_{0}\right)$. With no loss of generality we take the corner 0 to be at the origin, and assume that the two curves $\Gamma_{1}$ and $\Gamma_{2}$ forming the angle are $x_{2}=f_{1}\left(x_{1}\right)$ and $x_{1}=f_{2}\left(x_{2}\right)$ where $f_{i}\left(x_{i}\right) \in C_{m+2+\alpha}\left(\Gamma_{i}\right)$ and $f_{2}(0)=$ $f_{1}(0)=f_{1}^{\prime}(0)=0$. Consider the following transformation

$$
\begin{aligned}
& y_{1}=\frac{1}{\Delta \sqrt{ } \alpha_{11}}\left[\alpha_{12}\left(x_{1}-f_{2}\left(x_{2}\right)\right)+\alpha_{11}\left(x_{2}-f_{1}\left(x_{1}\right)\right)\right] \\
& y_{2}=\frac{1}{\sqrt{ } \alpha_{11}}\left[x_{1}-f_{2}\left(x_{2}\right)\right]
\end{aligned}
$$

where

$$
\begin{aligned}
\alpha_{11} & =a_{11}(0,0)-2 f_{2}^{\prime}(0) a_{12}(0,0)+f_{2}^{\prime 2}(0) a_{22}(0,0) \\
\alpha_{12} & =a_{22}(0,0) f_{2}^{\prime}(0)-a_{12}(0,0) \\
\alpha_{22} & =a_{22}(0,0) \\
\Delta & =\left[a_{11}(0,0) a_{22}(0,0)-a_{12}^{2}(0,0)\right]^{1 / 2}
\end{aligned}
$$


The two curves $\Gamma_{2}$ and $\Gamma_{1}$ will be transformed to $y_{2}=0$ and $y_{2}=y_{1} \tan \omega$, where $\tan \omega=\Delta / \alpha_{12},(\pi / \omega)>m+2+\alpha$. We now choose $\beta>0$ such that $\pi /(\omega+2 \beta)>$ $m+2+\alpha$, then we rotate the axes with this angle using the transformation

$$
\begin{aligned}
& z_{1}=y_{1} \cos \beta-y_{2} \sin \beta \\
& z_{2}=y_{1} \sin \beta+y_{2} \cos \beta .
\end{aligned}
$$

Consider the subdomain $G_{2}$ in $\bar{G}$ defined by

$$
G_{2}=\left\{\left(x_{1}, x_{2}\right):\left(x_{1}, x_{2}\right) \in \bar{G}, x_{1}^{2}+x_{2}^{2} \leq d_{1}^{2}, d_{1}>0\right\} .
$$

In the $z$-plane the domain $G_{2}$ will be transformed by (3.5)-(3.6) to a domain $\Omega_{0}$ bounded by the two lines $\theta=\beta$ and $\theta=\omega+\beta$ and by a curve $r=\sigma(\theta)$, where $\sigma(\theta) \geq \sigma>0, \quad \beta \leq \theta \leq \omega+\beta\left(r^{2}=z_{1}^{2}+z_{2}^{2}, \theta=\arctan z_{2} / z_{1}\right)$. In $\Omega_{\sigma}=$ $\left\{(r, \theta) \in \Omega_{0}, r \leq \sigma, \beta<\theta<\omega+\beta\right\}$ the transformed function $U(z)=U\left(z_{1}, z_{2}\right)=$ $u\left(x_{1}, x_{2}\right)$ satisfies the elliptic equation

$$
L U \equiv \sum_{i, j=1}^{2} b_{i j}(z) \frac{\partial^{2} U}{\partial z_{i} \partial z_{j}}+\sum_{i=1}^{2} b_{i}(z) \frac{\partial \bar{U}}{\partial z_{i}}+b(z) U=F(z)
$$

Where

$$
\begin{aligned}
& b_{11}(z)= e_{11}(z) \cos ^{2} \beta-2 e_{12}(z) \sin \beta \cos \beta+e_{22}(z) \sin ^{2} \beta \\
& b_{12}(z)= e_{11}(z) \sin \beta \cos \beta+e_{12}(z) \cos 2 \beta-e_{22}(z) \sin \beta \cos \beta \\
& b_{22}(z)= e_{11}(z) \sin ^{2} \beta+2 e_{12}(z) \sin \beta \cos \beta+e_{22}(z) \cos ^{2} \beta \\
& b_{1}(z)= e_{1}(z) \cos \beta-e_{2}(z) \sin \beta \\
& b_{2}(z)= e_{1}(z) \sin \beta+e_{2}(z) \cos \beta \\
& b(z)= a(x) \\
& F(z)= f(x) \\
& e_{11}(z)= \frac{1}{\Delta^{2} \alpha_{11}}\left[\left(\alpha_{11} f_{1}^{\prime}\left(x_{1}\right)-\alpha_{12}\right)^{2} a_{11}(x)\right. \\
&-2\left(\alpha_{11} f_{1}^{\prime}\left(x_{1}\right)-\alpha_{12}\right)\left(\alpha_{11}-\alpha_{12} f_{2}^{\prime}\left(x_{2}\right)\right) a_{12}(x) \\
&\left.+\left(\alpha_{11}-\alpha_{12} f_{2}^{\prime}\left(x_{2}\right)\right)^{2} a_{22}(x)\right] \\
& e_{12}(z)= \frac{1}{\Delta \alpha_{11}}\left[\left(\alpha_{12}-\alpha_{11} f_{1}^{\prime}\left(x_{1}\right)\right) a_{11}(x)\right. \\
&+\left(\alpha_{11}+\alpha_{11} f_{1}^{\prime}\left(x_{1}\right) f_{2}^{\prime}\left(x_{2}\right)-2 \alpha_{12} f_{2}^{\prime}\left(x_{2}\right)\right) a_{12}(x) \\
&\left.-f_{2}^{\prime}\left(x_{2}\right)\left(\alpha_{11}-\alpha_{12} f_{2}^{\prime}\left(x_{2}\right)\right) a_{22}(x)\right] \\
& \frac{1}{\alpha_{11}}\left[a_{11}(x)-2 f_{2}^{\prime}\left(x_{2}\right) a_{12}(x)+f_{2}^{\prime 2}\left(x_{2}\right) a_{22}(x)\right] \\
& e_{22}(z)=
\end{aligned}
$$




$$
\begin{aligned}
& \begin{aligned}
e_{1}= & \frac{1}{\Delta \sqrt{ } \alpha_{11}}\left[a_{1}(x)\left(\alpha_{12}-\alpha_{11} f_{1}^{\prime}\left(x_{1}\right)\right)+a_{2}(x)\left(\alpha_{11}-\alpha_{12} f_{2}^{\prime}\left(x_{2}\right)\right.\right. \\
& \left.-\alpha_{11} f_{1}^{\prime \prime}\left(x_{1}\right) a_{11}(x)-\alpha_{12} f_{2}^{\prime \prime}\left(x_{2}\right) a_{22}(x)\right] \\
e_{2}= & \frac{1}{\sqrt{\alpha_{11}}}\left[a_{1}(x)-f_{2}^{\prime}\left(x_{2}\right) a_{2}(x)-f_{2}^{\circ}\left(x_{2}\right) a_{22}(x)\right]
\end{aligned} \\
& b_{11}(z) b_{22}(z)-b_{12}^{2}(z)=\frac{\sin ^{4} \beta+\cos ^{4} \beta}{\Delta^{2}}\left[1-f_{1}^{\prime}\left(x_{1}\right) f_{2}^{\prime}\left(x_{2}\right)\right]^{2}\left[a_{11}(x) a_{22}(x)-a_{12}^{2}(x)\right]
\end{aligned}
$$

The functions $b_{i j}, b_{i}, b$ and $F$ belong to $C_{m+\alpha}\left(\bar{\Omega}_{\sigma}\right)$ and $b_{i j}(0,0)=\delta_{i j} i, j=1,2$. On the two lines $\theta=\beta$ and $\theta=\omega+\beta$ the boundary values $\Phi(r, \beta)$ and $\Phi(r, \omega+\beta)$ of $U(z)$ belong to $C_{m+2+\alpha}, 0 \leq r \leq \sigma$ and $\Phi(0, \beta)=\Phi(0, \omega+\beta)$. We denote by $\Phi^{0}$ and $\Phi_{\beta}^{k}$ the values of $\Phi(r, \beta)$ and $\left(d^{k} \Phi / d r^{k}\right)(r, \beta)$ at $r=0$, $k=1,2, \ldots, m+2 . \Phi_{\omega+\beta}^{k}$ is defined similarly. Consider now the function

$$
\begin{aligned}
& \Theta(z)=\Phi^{0}+\sum_{k=1}^{m+2} \frac{\left(z_{1} \cos \beta+z_{2} \sin \beta\right)^{k}}{k !} \Phi_{\beta}^{k} \\
& +\sum_{k=1}^{m+2} \sum_{l=1}^{k} \frac{\left(z_{1} \cos \beta+z_{2} \sin \beta\right)^{k-l}\left(-z_{1} \sin \beta+z_{2} \cos \beta\right)^{l}}{(k-l) ! l !} \cdot \frac{\Phi_{\omega+\beta}^{k}-\Phi_{\beta}^{k} \cos ^{k} \omega}{(\sin \omega+\cos \omega)^{k}-\cos ^{k} \omega} .
\end{aligned}
$$

The function $v(z)=U(z)-\Theta(z)$ satisfies in $\Omega_{\sigma}$ the equation

$$
L v=h(z) \equiv F-L \Theta(z) \text {. }
$$

On the two lines $\theta=\beta$ and $\theta=\omega+\beta$ the boundary value $\Psi(r, \theta)$ of $v(z)$ belongs to $C_{m+2+\alpha}$ and satisfies (2.2). In Lemma 3 we will show that there exists a polynomial $s(z)=s\left(z_{1}, z_{2}\right)$ vanishing on $\theta=\beta$ and $\theta=\omega+\beta$ such that $w=v-s$ satisfies in $\Omega_{\sigma}$ equation (2.4) with $g(z)=h(z)-L s(z)$ satisfying (2.3). Thus all the conditions of Theorem 1 are satisfied, and we conclude that in $\bar{\Omega}_{r_{0}}$, $2 r_{0}<\sigma, w(z) \in C_{m+2+\alpha}$. Since $U(z)=w(z)+\Theta(z)+s(z)$, where $\Theta(z)$ and $s(z)$ are polynomials, then $U(z) \in C_{m+2+\alpha}\left(\bar{\Omega}_{r_{0}}\right)$. Returning now to the $x$-plane and noting that the transformation (3.5) is of class $C_{m+2+\alpha}$, and at the origin it has a Jacobian equal to $-(1 / \Delta)$ which is finite and different from zero, we conclude that in a subdomian $\bar{G}_{0} \subset \bar{G} ; G_{0}=\left\{\left(x_{1}, x_{2}\right):\left(x_{1}, x_{2}\right) \in \bar{G}, x_{1}^{2}+x_{2}^{2} \geq d_{2}^{2}, d_{2}>0\right\}$, $u\left(x_{1}, x_{2}\right) \in C_{m+2+\alpha}$. This proves the theorem.

LEMMA 3. There exists a polynomial $s(z)$ vanishing on the two lines $\theta=\beta$ and $\theta=\omega+\beta$, and is such that the function $w(z)=v(z)-s(z)$ satisfies in $\Omega_{\sigma}$ the equation $L w=g(z) \equiv h(z)-L s(z)$, with $g(z)$ satisfying (2.3).

Proof. We set $m_{1}=\tan \beta$ and $m_{2}=\tan (\omega+\beta), 1+m_{1} m_{2} \neq 0$ and by $h^{\left(k_{1}, k_{2}\right)}(0,0)$ we denote

$$
\left.\frac{\partial^{k_{1}+k_{2} h}}{\partial x_{1}^{k_{1}} \partial x_{2}^{h_{2}}}\right|_{(0,0)}, \quad 1 \leq k_{1}+k_{2} \leq m .
$$


We will prove the lemma by induction. Consider the function

$$
s_{0}(z)=\left(z_{2}-m_{1} z_{1}\right)\left(z_{2}-m_{2} z_{1}\right) \frac{h(0,0)}{2\left(1+m_{1} m_{2}\right)}
$$

$s_{0}(z)$ vanishes on $\theta=\beta$ and $\theta=\omega+\beta$, and

$$
L s_{0}(z)=h(0,0)+p_{1}(z)+o(r)
$$

where $p_{k}(z)$ is a homogeneous polynomial in $z_{1}$ and $z_{2}$ of order $k, k=$ $1,2, \ldots, m$. Consider now the two functions

$$
\begin{aligned}
P_{k-1}(z) & =\left(z_{2}-m_{1} z_{1}\right)\left(z_{2}-m_{2} z_{1}\right) T_{k-1} \\
P_{k} & =\left(z_{2}-m_{1} z_{1}\right)\left(z_{2}-m_{2} z_{1}\right) T_{k}
\end{aligned}
$$

where

$$
\begin{aligned}
T_{k-1}(z) & =\sum_{k_{1}+k_{2}=0}^{k-1} \lambda_{k_{1} k_{2}} z_{1}^{k_{1}} z_{2}^{k_{2}} \\
T_{k}(z) & =\sum_{k_{1}=0}^{k} \lambda_{k_{1}} z_{1}^{k_{1}} z_{2}^{k-k_{1}}, \quad k \leq m
\end{aligned}
$$

We put

$$
s_{k}(z)=P_{k-1}(z)+P_{k}(z)
$$

Suppose now that the coefficients $\lambda_{k_{1} k_{2}}$ in $P_{k-1}(z)$ are already found such that

$$
L P_{k-1}(z)=\sum_{k_{1}+k_{2}=0}^{k-1} \frac{z_{1}^{k_{1}} z_{2}^{k_{2}}}{k_{1} ! k_{2} !} h^{\left(k_{1}, k_{2}\right)}(0,0)+p_{k}(z)+o\left(r^{k}\right)
$$

Now we find the coefficients $\lambda_{0}, \lambda_{1}, \ldots, \lambda_{k}$ in $P_{k}$ such that

$$
L P_{k}(z)=\sum_{k_{1}=0}^{k} \frac{z_{1}^{k_{1}} z_{2}^{k-k_{1}}}{k_{1} !\left(k-k_{1}\right) !} h^{\left(k_{1}, k-k_{1}\right)}(0,0)+p_{k+1}(z)-p_{k}(z)+0\left(r^{k+1}\right)
$$

where $p_{k}(z)$ is taken from (3.9). Now

$$
L P_{k}(z)=\sum_{i, j=1}^{2} b_{i j}(z) \frac{\partial^{2} P_{k}}{\partial z_{i} \partial z_{j}}+p_{k+1}(z)+o\left(r^{k+1}\right)
$$

and since $b_{i j}(0,0)=\delta_{i j}$ we get

$$
L P_{k}(z)=\frac{\partial^{2} P_{k}}{\partial z_{1}^{2}}+\frac{\partial^{2} P_{k}}{\partial z_{2}^{2}}+p_{k+1}(z)+o\left(r^{k+1}\right)
$$

We now find $\lambda=\left\{\lambda_{0}, \lambda_{1}, \ldots, \lambda_{k}\right\}$ from the identity

$$
\frac{\partial^{2} \boldsymbol{P}_{k}}{\partial z_{1}^{2}}+\frac{\partial^{2} \boldsymbol{P}_{k}}{\partial z_{2}^{2}}=\sum_{k_{1}=0}^{k} \frac{z_{1}^{k_{1}} z_{2}^{k-k_{1}}}{k_{1} !\left(k-k_{1}\right) !} h^{\left(k_{1}, k-k_{1}\right)}(0,0)-p_{k}(z)
$$

Equating the coefficients of $z_{1}^{k_{1}} z_{2}^{k-k_{1}}, k_{1}=0,1, \ldots, k$ on both sides of (3.11) 
we get the system of algebraic equations

$$
A \lambda=B
$$

If $\operatorname{det} A \neq 0$, then (3.12) is solvable for $\lambda$. We now show that $\operatorname{det} A \neq 0$. Suppose contrarily that $\operatorname{det} A=0$. We find a nonzero solution $\lambda^{*}$ of the equation

$$
A \lambda=0
$$

then we substitute this solution in $P_{k}(z) . P_{k}(z)$ is now a homogeneous polynomial of order $k+2$, vanishes on the two lines $z_{2}=z_{1} \tan \beta$ and $z_{2}=z_{1}$ $\tan (\omega+\beta)$ and satisfies in $\Omega_{\sigma}$ the Laplace equation

$$
\frac{\partial^{2} P_{k}}{\partial z_{1}^{2}}+\frac{\partial^{2} P_{k}}{\partial z_{2}^{2}}=0 .
$$

Thus $P_{k}(z)$ vanishes on all the lines making angles $t \omega$ with these two lines, where $t$ is any positive integer. If $\pi / \omega$ is irrational, then the number of these lines is not finite. If $\omega=(p / q) \pi$ where $p / q$ is an irreducible fraction and $p \geq 1$, then the number of these lines is $q, q \geq(q / p)=(\pi / \omega)>m+2+\alpha$. In both cases the number of the different lines on which $P_{k}(z)$ vanishes is greater than $m+2$. This is a contradiction since $P_{k}(z)$ is a polynomial of degree $k+2, k \leq m$. Thus $\operatorname{det} A \neq 0$, and (3.12) uniquely gives $\lambda$. The function $s_{m}(z)$ satisfies all the requirements of the lemma. This proves the lemma.

\section{REMARKS.}

1. If $(\pi / \omega) \leq m+2+\alpha$, then using the same argument we can show that $u(x) \in C_{(\pi / \omega)-\varepsilon}(\bar{G}), \varepsilon>0$ is arbitrary.

2 . If there is more than one corner on the boundary then the smoothness of the solution in the neighborhood of each corner may be discussed separately.

Acknowledgement. The author wishes to express his gratitude to Professor V. A. Kondratev of the Moscow State University, for introducing him to the subject of this article, and for his helpful discussion during its preparation.

\section{REFERENCES}

1. S. Agmon, A. Douglis, L. Nirenberg, Estimates near the boundary for solutions of elliptic partial differential equations, satisfying general boundary conditions. Comm. Pure Appl. Math N 12 (1959), 623-727.

2. F. Browder, Estimates and existence theorems for elliptic boundary value problems. Proc. Nat. Acad. Sci. U.S.A. 45 (1959), 365-372.

3. V. V. Fufaev, On the Dirichlet problem for regions with corners, Dolk, Akad. Nauk SSSR 131 (1960), 37-39.

4. O. D. Kellog, On the derivatives of harmonic functions on the boundary. Trans. Amer. Math. Soc. 33 (1931), 486-510. 
5. V. A. Kondratev, Boundary value problems for elliptic equations in domains with conical or angular points. Tran. of the Moscow Mat. Soc. 16 (1967), 227-313.

6. V. A. Kondratev, The smoothness of a solution of Dirichlet's problem for second order elliptic equations in a region with a piecewise smooth boundary. Differentsialnye Uravneneya 10 (1970), 1831-1843.

7. O. A. Ladyzhenskaya, Ural'tseva, N. N., Linear and quasilinear elliptic equations. Academic Press, New York and London 1968.

8. R. Sherman Lehman, Developments at an analytic corner of the solutions of elliptic partial differential equations. J. Math. Mech. 8 (1959), 727-760.

9. H. Lewy, Developments at the confluence of analytic boundary conditions. Univ. California Publ. Math. (N.S.) 1, (1950) 247-280.

10. C. Miranda, Partial differential equations of elliptic type. Springer-Verlag New York 1970.

11. S. M. Nikolskii, Harmonic functions on rectangular regions. Mat. Sbornik (N.S.), 43 (85) (1957), 127-144.

12. S. L. Sobolev, Applications of functional analysis in mathematical physics. Providence, A.M.S., 1963.

13. E. A. Volkov, On the differentiability properties of solutions of boundary value problems for the Laplace and Poisson equations on a rectangle. Trudy Mat. Inst. Steklov, 77 (1965), 89-112.

14. - Differential properties of solutions of boundary value problems for the Laplace equation on polygons. Trudy Mat. Inst. Steklov, 77 (1965), 113-142.

15. W. Wasow, Asymptotic development of the solution of Dirichlet's problem at analytic corners. Duke Math. J. 24 (1957), 47-56.

16. Neil M. Wigley, Asymptotic expansion at a corner of solutions of mixed boundary value problems. J. Math. Mech. 13 (1964), 549-576.

17. - Mixed boundary value problems in plane domains with corners. Math. Z. 115 (1970) 33-52.

DePARTMENT OF MATHEMATICS

UNIVERSITY OF WINDSOR

WINDSOR, ONT. N9B 3P4 